\title{
Abordagem de recomendações de design da Interação Criança-Computador no curso de formação de professores em uma linguagem de programação visual em blocos
}

\author{
Esteic Janaina Santos Batista ${ }^{1,2}$, Camila Leite da Silva ${ }^{1,2}$, Anderson Corrêa de \\ Lima $^{1}$ \\ ${ }^{1}$ Universidade Federal de Mato Grosso do Sul campus Ponta Porã (UFMS/CPPP) - \\ Ponta Porã - MS - Brasil \\ ${ }^{2}$ Bolsistas do Programa de Educação Tutorial - PET/SESu/MEC \\ \{esteicejanaina, camila.leite002, anderson.correa.lima\}@gmail.com
}

\begin{abstract}
This study analyzes games developed in visual block programming language from the final project of a Scratch course for teachers. The analysis of the games turned in to verify if the games developed by these teachers were adapted for the age group of the public to which all children were destined. For the analysis of these games were taken into consideration some elements of design, and, as well, K-12 education children evaluation. . It also discusses the importance of a teacher-training course that presents these recommendations, contributing to the fact that educational objects more effectively fulfill their goals in the classroom.
\end{abstract}

Resumo. Este estudo analisa jogos desenvolvidos na linguagem de programação visual em blocos oriundos de projetos finais de um curso de Scratch para professores. A análise dos jogos voltou-se em verificar se os jogos desenvolvidos por estes professores estavam adequados para a faixa etária do público a que se destinava, sendo, na sua totalidade, crianças. Para $a$ análise destes jogos foram levados em consideração alguns elementos de design, e, também, a avaliação por crianças do ensino fundamental. Discutese ainda, a importância de um curso de formação de professores que apresente essas recomendações, contribuindo para que os objetos educacionais cumpram, de forma mais efetiva, seus objetivos em sala de aula.

\section{Introdução}

As aplicações voltadas para o público infantil tornam-se cada vez mais crescentes, e é frequente e diversificada sua utilização por professores em sala de aula, seja através da utilização ou desenvolvimento de seus próprios objetos de aprendizagem.

O termo de IHC (Interação Humano-Computador) foi definido como um campo de estudo com interesse nos aspectos relacionados à interação entre usuários e computadores, onde o design de interação é um dos temas de estudo [Bezerra 2010]. O design de interação refere-se à criação de produtos centrados nos utilizadores, levando em consideração seus objetivos, necessidades, habilidades, características e experiências, para que resultem em interfaces que sejam eficazes, eficientes, fáceis de 
VI Congresso Brasileiro de Informática na Educação (CBIE 2017)

Anais do XXIII Workshop de Informática na Escola (WIE 2017)

manipular, agradáveis e motivadoras, para assim atingir as metas de usabilidade [Preece et al. 2005].

No entanto, quando o usuário é uma criança, fica mais difícil compreender como ela interage com os dispositivos [Mano 2005], sejam eles computadores, celulares, tablets, e, até mesmo, em ambientes de total imersão visual, como é o caso da Realidade Virtual e Aumentada. Essa dificuldade se deve ao fato de que as crianças apresentam muitas peculiaridades que diferem dos usuários adultos, tais como aquelas relacionadas com a idade e a percepção.

De tal forma, uma subárea da Interação Humano-Computador é definida, sendo essa o campo da Interação Criança-Computador (ICC), que diz respeito ao estudo da concepção, avaliação e implementação de sistemas computacionais interativos para crianças, analisando o impacto mais amplo da ação da tecnologia nestas e na sociedade [Hourcade 2015].

O campo de IHC, bem como o de desenvolvimento de jogos, deixou de ser destinado apenas para pessoas com formação em computação. As linguagens de programação visual em blocos, cada vez em maior destaque no meio educacional, permite que estudantes do ensino básico e professores criem seus próprios jogos e objetos de aprendizagem [Batista e Castro 2016].

Este trabalho analisa alguns jogos educacionais desenvolvidos por professores em seus projetos finais do curso de formação de professores na linguagem de programação visual em blocos utilizando a ferramenta Scratch. Procurou-se verificar a presença de recomendações de design da Interação Criança-Computador nos jogos desenvolvidos, sendo consideradas as recomendações dos seguintes elementos do design de interação: affordance, feedback, navegação, gestos, sons, cores, tipografia, ícones e personagens.

Os jogos foram analisados por crianças já conhecedoras da ferramenta Scratch, e, para a análise foram apresentadas frases simplificadas correspondendo a cada item de design. As crianças utilizaram a escala Likert, por meio de uma avaliação com smileys, ao invés de pontuação.

Este trabalho está estrutura da seguinte forma: a Seção 2 apresenta sobre o curso de formação de professores para uso da ferramenta de programação visual Scratch; a Seção 3 apresenta sobre a Interação Criança-Computador e os elementos de design que foi também utilizado na avaliação dos jogos desenvolvidos no Scratch; a Seção 4 apresenta a metodologia empregada na avaliação dos jogos e quais os jogos selecionados para este estudo; a Seção 5 discute os resultados da avaliação e levanta alguns elementos que poderiam ser adicionados em um jogo ou objeto de aprendizagem desenvolvido no Scratch, dentro das possibilidades da ferramenta; e por fim, nas considerações finais apresentaremos uma discussão sobre a importância de um curso auxiliar no campo de ICC na formação de professores.

\section{Formação de professores com o Scratch}

O Scratch é um projeto do grupo Lifelong Kindergarten no Media Lab do Instituto de Tecnologia de Massachusetts (MIT) dos Estados Unidos. Trata-se de uma ferramenta de 
programação visual em blocos, consistindo, atualmente, de duas principais versões: o Scratch 2, que é disponibilizado para desktop e online, para crianças a partir de 8 anos com habilidade de leitura, e o ScratchJr, para crianças a partir de 4 anos, e disponibilizado para dispositivos móveis touchscreen.

A ferramenta possui códigos simples para comandar o personagem por blocos, que permitem ser encaixados, para criação de uma aplicação, remetendo os brinquedos de encaixe Lego, baseado no conceito de drag-and-drop (arrastar e largar). Deste modo, é possível criar jogos, animações e histórias, em um ambiente intuitivo, divertido e fácil, possibilitando que o usuário altere o cenário, personagens, inclua sons, utilize a câmera, permitindo ser usado nas mais diferentes disciplinas [Batista, Castro Jr., Cantero, Bogarim e Larrea 2016].

Ele vem sendo utilizado nas escolas, e permitindo seu uso de duas principais maneiras: seja o professor propondo desafios para os alunos desenvolverem em aula, através da criação de jogos e histórias na temática do assunto abordado, ou o professor desenvolvendo seus próprios jogos e objetos de aprendizagem para aplicar em suas aulas.

Foi ofertado um curso de Scratch aos Professores Gerenciadores da Sala de Tecnologia (PROGETEC's), que são responsáveis por oferecer capacitações sobre o uso das Tecnologias da Informação e Comunicação (TICs) aos professores e gerenciar a sala de tecnologia da escola onde atuam. Durante o curso foram propostas diversas atividades para que os cursistas explorassem os recursos da ferramenta, e, o projeto final consistia em que o professor desenvolvesse um jogo como objeto educacional [Batista e Castro 2016].

Foram avaliados os projetos finais de uma turma, totalizada em trinta cursistas. Verificou-se que alguns jogos não estavam adequados em termos de usabilidade para o público final ao qual se destinava.

Uma vez que os professores assumem papel de desenvolvedores, torna-se também necessário que haja a preocupação em entender alguns elementos de design, para que seu objeto de aprendizagem alcance os objetivos de usabilidade, tornando assim, suas produções mais atrativas aos estudantes, e, consequentemente, atingindo seu objetivo na aula.

\section{Recomendações de Design da Interação Criança-Computador}

As recomendações de design que serão analisadas neste estudo basearam-se em alguns trabalhos centrais. Para a escolha dos elementos chaves que analisaremos, nos baseamos no trabalho de [Fabossi e Guimarães 2014] e [Sesame Workshop 2012] que trazem premissas de design de interação e apresentam boas práticas para projetar aplicações destinadas a crianças. Já o trabalho de [Resende 2010] levanta diversas recomendações de usabilidade para interfaces e design para crianças de 6 a 10 anos.

No trabalho de [Mano 2005] foi realizada a construção de interfaces de computador para crianças, identificando o que pode ou não resultar numa interface humano-computador para crianças entre os 5 a 7 anos, e de que forma crianças desta idade reagem perante a interface. Desta forma, tentou-se estabelecer um conjunto de 
guias para a criação de um software infantil e didático.

Neste trabalho, buscou-se delimitar um conjunto de elementos guias para o design infantil. Os elementos de design selecionados e que foram utilizados para avaliar os jogos são: affordance, feedback, navegação, gestos, sons, cores, tipografia e personagens; eles são apresentados de forma breve nas subseções seguintes.

\subsection{Affordances}

Affordances são características de objetos capazes de revelar a seus usuários as suas operações e manipulações. Ainda, segundo [Bezerra 2010] refere-se ao "atributo de um objeto que permite às pessoas saber como utilizá-lo", é dar uma dica de como funciona sua operação. Em uma interface, elementos como: botões e ícones devem por si só demonstrar qual a sua função.

Para o público infantil, a [Sesame Workshop 2012] sugere o uso de ícones consistentes e representacionais que seguem a convenção padrão, e, sugere também, indicações explícitas na tela para indicar quando a criança pode interagir. A affordance dá aos elementos interativos a característica de serem clicáveis. Ela facilita para que o usuário aprenda e memorize com mais facilidade as ações do sistema.

\subsection{Feedbacks}

O Feedback refere-se à resposta de uma ação realizada, permitindo ao usuário continuar a atividade, podendo ser: auditivo, tátil, visual, verbal, ou combinado [Bezerra 2010]. O processo de fornecer respostas adequadamente é fundamental para proporcionar uma boa experiência para as crianças. A resposta errada é encarada como uma oportunidade para um momento de aprendizagem, e, nestas situações, precisa-se empregar de forma encorajadora um feedback visual e auditivo. Os incentivos são muito importantes, pois mantêm os pequenos usuários motivados [Fabossi e Guimarães 2014].

As crianças esperam ver os resultados de suas ações imediatamente, "mostrando que suas ações tiveram algum efeito, caso contrário, elas irão repetir a ação até algo ser percebido. Possivelmente, tal comportamento causará uma sequência de eventos inesperados e indesejados" [Bezerra 2010].

É recomendado o feedback no início de um jogo para guiar as crianças no aprendizado de novos conceitos, porém, a remoção gradual do feedback é recomendável para o estímulo desse aprendizado em fases mais avançadas [Bezerra 2010].

O estudo da [Sesame Workshop 2012] recomenda que o uso de feedback auditivo e visual deve ser encorajador e incremental, e, define ainda, estágios de feedback para respostas incorretas do usuário. Para a resposta incorreta dada pelo usuário na primeira vez, a frase deve informá-lo e encorajá-lo. Exemplo: "Não é isso. Tente novamente!". No segundo erro consecutivo, deve-se além disso, oferecer uma dica e encorajá-lo. No terceiro erro consecutivo da questão, acrescenta-se uma reafirmação do objetivo, oferecendo uma dica e destacando a resposta correta.

Quando as respostas estiverem corretas, é importante recompensar as crianças, pois isso as mantêm motivadas.

\subsection{Navegação}


As interfaces de aplicativos para as crianças precisam ser intuitivas e consistentes, pois são mais fáceis de serem aprendidas e usadas. Elas seguem regras, como exemplo, uma mesma ação (modo de operação) para seleção de objetos, assim, funções similares devem possuir elementos semelhantes e serem agrupadas, fortalecendo a consistência e a continuidade de uma interface [Fabossi e Guimarães 2014].

Segundo [Bezerra 2010] "as interfaces consistentes são mais fáceis de aprender e de usar, pois os usuários só precisam aprender um único modo de operação aplicável a todos os objetos". O [Sesame Workshop 2012] sugere a utilização de um tempo limite com uma sugestão concisa para o que fazer em seguida. Ainda é proposto que os objetivos dos jogos estejam visualmente explícitos, e sejam imediatos e intuitivamente óbvios em cada tela.

\subsection{Gestos}

Uma interface simplificada poderá facilitar a operação com o mouse, e, as interfaces para crianças devem incluir áreas clicáveis grandes o bastante para compensar alguma falta de habilidade no manuseio do mouse. Além disso, tais áreas devem estar dispostas com espaços entre elas, de modo a diminuir as chances de a criança pressionar uma área errada [Bezerra 2010].

\subsection{Sons}

Os sons em aplicações destinadas a crianças desempenham um papel fundamental. Segundo [Fabossi e Guimarães 2014] "crianças preferem ouvir, considerando também que este ato exige menos esforço cognitivo. Os sons podem transmitir estados emocionais, bem como suscitar respostas emocionais aos usuários".

De acordo com [Sesame Workshop 2012] o som precisa ser conciso, pois as crianças normalmente não prestam atenção apenas às instruções de áudio, logo, é preciso manter um áudio essencial mínimo e preciso. Sugere-se ainda que as instruções de áudio ininterruptas e mensagens não essenciais possam ser interrompidas, especialmente na repetição, pois isso deixa as crianças impacientes.

A [Sesame Workshop 2012] define ainda que os sons podem ser utilizados como feedbacks imediatos às ações do usuário. E que, quando possível, as ações do usuário devam ser reconhecidas com um som correspondente. Os fundos musicais podem melhorar o engajamento para uma criança, mas é necessário monitorar o volume da música de fundo.

\subsection{Cores}

As cores em aplicações destinadas a crianças desempenham um papel fundamental, segundo [Resende 2010], esta é a primeira coisa a ser percepcionada pela criança ao entrar na interface. Cores vibrantes chamam atenção das crianças, e suas preferidas são vermelho e laranja, segundo as conclusões da pesquisa relatada em seu trabalho. A escolha das cores na construção de uma interface, principalmente no agrupamento de cores, deve ser feito com cautela, pois uma escolha não adequada de cores pode interferir na legibilidade da interface [Resende 2010]. É recomendável utilizar mais de uma cor no mesmo aplicativo [Sesame Workshop 2012]. 


\subsection{Tipografia}

Quanto mais nova for a criança, menos texto deve ser utilizado na aplicação, devido às habilidades de leitura que serão aprimoradas no decorrer do desenvolvimento da criança na infância. Especificamente para as crianças da educação infantil, as instruções primárias e outras instruções devem ser transmitidas de forma verbal e visual, para que assim elas possam ter uma boa experiência na utilização da aplicação, sem a ajuda dos pais. [Sesame Workshop 2012].

\subsection{Personagens}

O personagem tem importância relevante na experiência de uso de um sistema para crianças mais novas, pois eles possuem a capacidade de atrair a atenção de forma lúdica, e de comunicar uma mensagem com facilidade, participação e alegria [Fabossi e Guimarães 2014].

De acordo com [Sesame Workshop 2012], os personagens podem assumir o papel de "guias" ao longo do processo de aprendizagem, e trazem para as crianças um sentimento de amizade e diversão, que é fundamental para construir um relacionamento em experiências de mídia digital. Isso cria um ambiente de engajamento e diálogo entre a criança e o personagem na tela. É dito ainda que a maior parte do conteúdo começa com um personagem ou um narrador adulto amigável saudando o usuário.

\section{Avaliação dos jogos}

Foram escolhidos seis jogos desenvolvidos pelos professores do curso de Scratch para análise neste trabalho. Para a escolha foram levados em consideração os que tinham objetivos de aprendizagem mais claros, e maior número de elementos de um jogo. Foram analisados os elementos de design descritos na seção anterior, sendo eles: affordance, feedback, navegação, gestos, sons, cores, tipografia e personagens. As interfaces dos jogos são apresentadas na Tabela 1. Segue uma breve descrição de cada um dos jogos:

a) Pacman contra a sujeira: o jogo consiste em um labirinto onde o personagem deverá recolher os lixos. O objetivo foi conscientizar as crianças sobre $\mathrm{o}$ descarte correto do lixo.

b) Labirinto saudável: o jogo consiste em levar o gato até os alimentos saudáveis. O objetivo é o jogador testar seus conhecimentos sobre alimentação.

c) Capitais: o objetivo do jogo é o usuário responder uma série de perguntas sobre as capitais dos estados brasileiros.

d) Jogos dos 5 erros no combate contra a Dengue: o usuário deve, neste jogo, clicar nos itens que podem acumular água, aumentando as chances de criação do mosquito da dengue.

e) GeoBrink - brincando com os sólidos geométricos: o jogo explica sobre os sólidos geométricos e os usuários podem testar seus conhecimentos respondendo a algumas perguntas sobre os sólidos geométricos apresentados. 
VI Congresso Brasileiro de Informática na Educação (CBIE 2017)

Anais do XXIII Workshop de Informática na Escola (WIE 2017)

f) “Dengue, aqui não!”: o jogo apresenta informações sobre a Dengue e o usuário pode responder um quiz para teste de seus conhecimentos.

Tabela 1. Jogos escolhidos para a avaliação

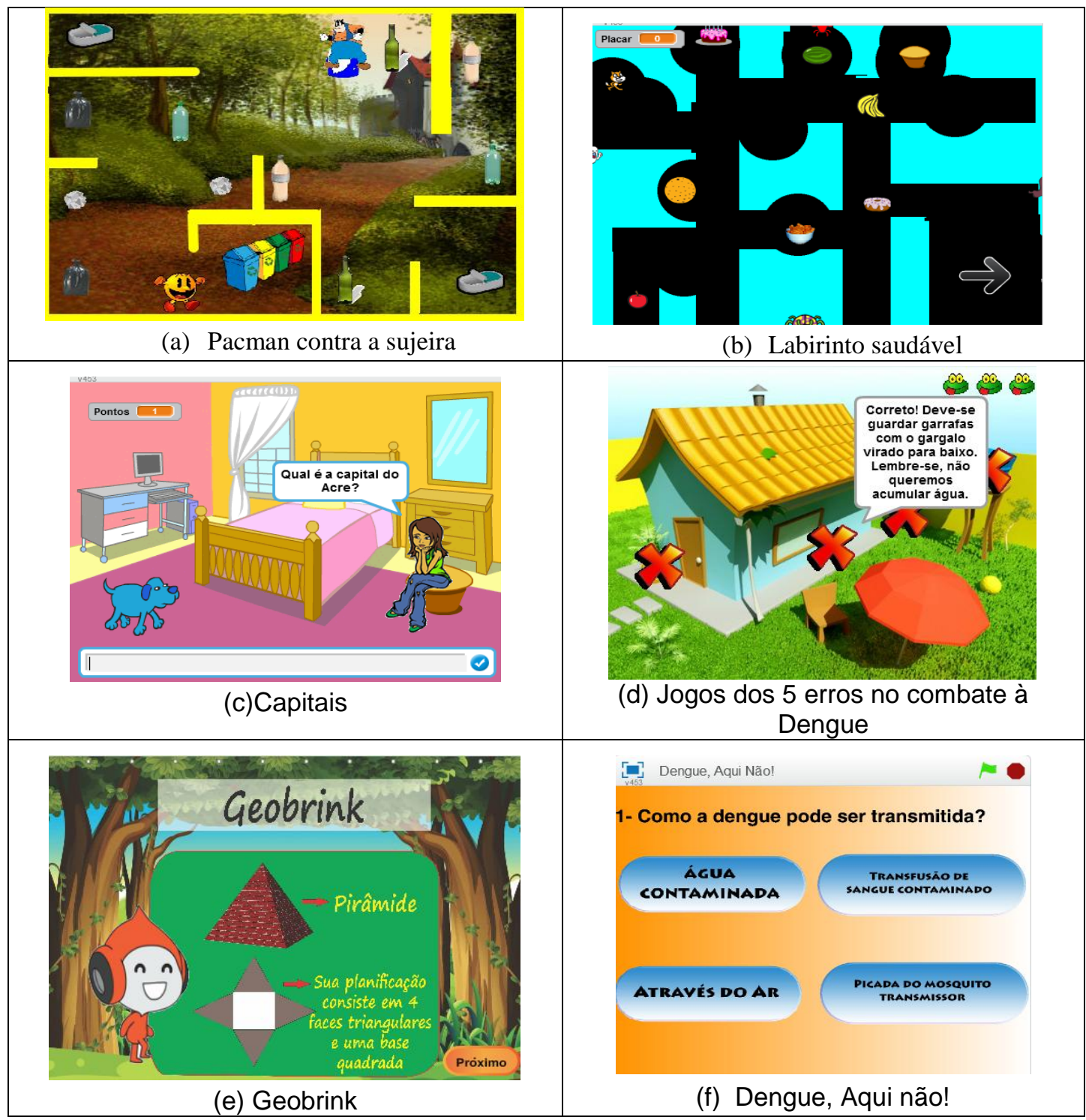

Para a análise de cada um dos elementos de design, foi utilizada a escala do tipo Likert de cinco pontos [Andrade 2015] para descrição quantitativa dos indicadores, por apresentar-se mais adequada em relação à precisão e confiabilidade apresentados no estudo de [Dalmoro e Vieira 2013]. A escala foi adaptada para crianças, com o objetivo de identificar como a criança está se sentindo em relação ao que foi proposto utilizando o Smileyometer (Figura 1).

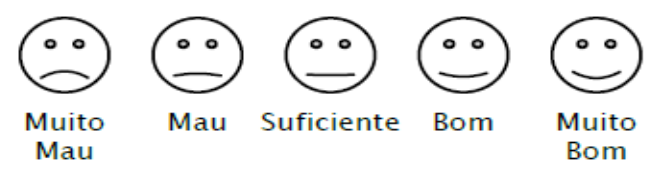

Figura 1. Smileyometer [Valente 2011] 
VI Congresso Brasileiro de Informática na Educação (CBIE 2017)

Anais do XXIII Workshop de Informática na Escola (WIE 2017)

No total, cinco crianças do $5^{\circ}$ ao $9^{\circ}$ ano do ensino fundamental avaliaram os jogos em uma tabela com as questões simplificadas (Figura 2). As crianças que avaliaram os jogos já conheciam a ferramenta Scratch, e já haviam desenvolvido jogos no ambiente.

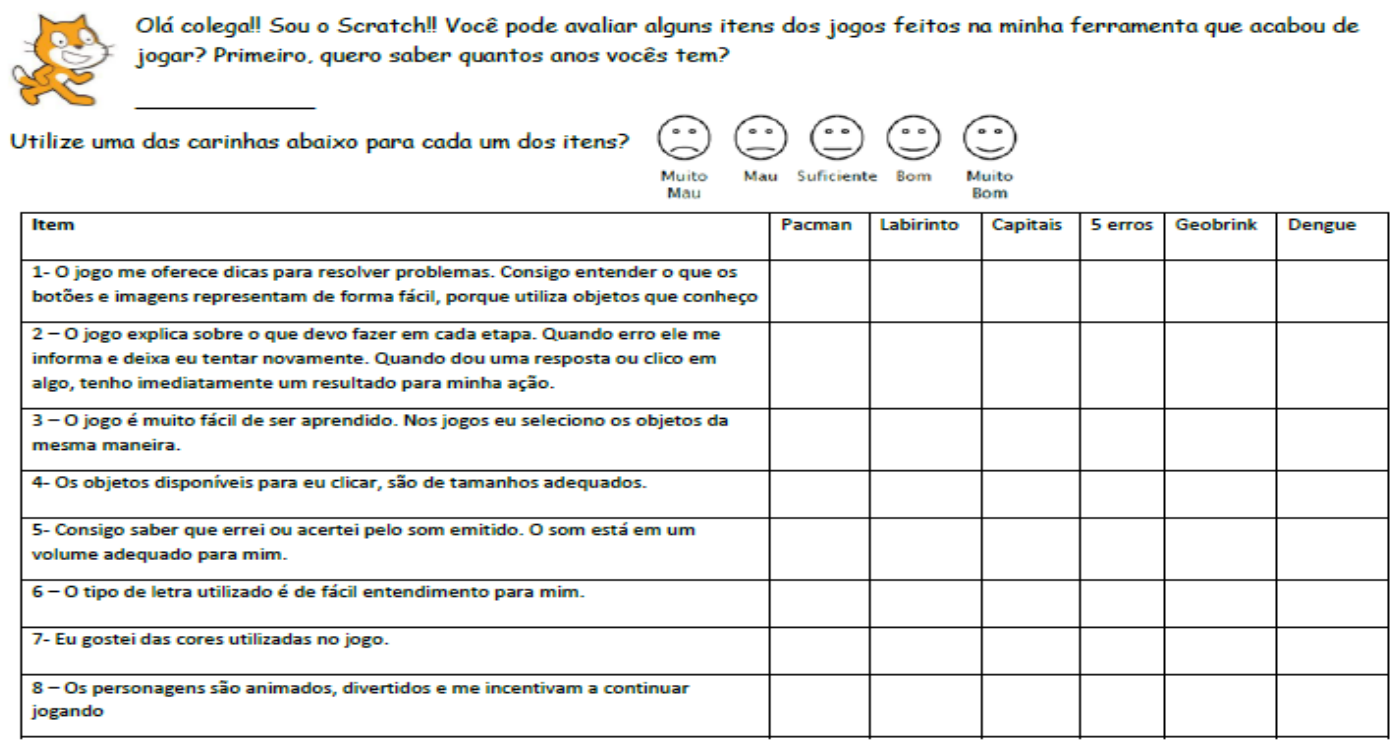

Figura 2. Perguntas simplificadas correspondentes a cada item de design

\section{Análise e discussão dos resultados}

Na Tabela 2 é apresentada a média das avaliações de cada item de design para cada um dos jogos. Foi atribuído do valor 1 (muito mau do Smiley) a 5 (muito bom do Smiley). Foram inseridos apenas o termo do elemento de design correspondente, de forma a facilitar a interpretação.

A partir da análise dos jogos observou-se que as crianças apontaram de forma mediana o feedback apresentado. Nos jogos do Labirinto e do Pacman, observou-se que a primeira tela já consistia no próprio jogo, e não havia um personagem ou um cenário que explicasse sobre os comandos que deveriam ser utilizados, e nem regras de pontuação. Em outros jogos, como o da Dengue e o de capitais, o feedback não permitia que o usuário tivesse outra chance de acertar a questão. Os sensores poderiam ter sido utilizados com maior frequência, de forma a personalizar o jogo para o usuário.

Os affordances, de forma geral, praticamente não foram utilizados pelos jogos desenvolvidos. Apareciam poucas vezes os personagens com papel importante, quando o jogo possuía um. Um exemplo é no jogo Geobrink em que o personagem não tinha ação quando era apresentado ao usuário o conteúdo de sólidos geométricos, podendo ter sido utilizadas mais animações nos personagens e nos próprios sólidos, para deixar o jogo mais interativo. Os sons foram utilizados em alguns jogos, porém, poderia ter sido explorada a utilização de diferentes sons nas trocas de cenário, por exemplo, quando o usuário saísse do ambiente de explicação para o ambiente de perguntas. $\mathrm{O}$ volume do som também estava inadequado, sendo interessante o controle de volume na programação, visto que a ferramenta de desenvolvimento oferece este recurso. 
VI Congresso Brasileiro de Informática na Educação (CBIE 2017)

Anais do XXIII Workshop de Informática na Escola (WIE 2017)

Tabela 2. Jogos escolhidos para a avaliação

\begin{tabular}{|l|c|c|c|c|c|c|}
\hline Item/Jogo & Pacman & Labirinto & Capitais & 5 erros & Geobrink & Dengue \\
\hline Affordance & 3 & 3 & 3 & 3 & 3 & 3 \\
\hline Feedback & 3 & 2 & 3 & 4 & 3 & 3 \\
\hline Navegação & 3 & 3 & 3 & 4 & 4 & 4 \\
\hline Gestos & 3 & 3 & 3 & 4 & 4 & 4 \\
\hline Sons & 3 & 2 & 2 & 4 & 2 & 4 \\
\hline Tipografia & 5 & 5 & 5 & 5 & 5 & 5 \\
\hline Cores & 5 & 4 & 4 & 4 & 4 & 4 \\
\hline Personagem & 3 & 3 & 2 & 4 & 4 & 4 \\
\hline
\end{tabular}

As carências apresentadas nos itens de design avaliados podem ser resolvidas com alterações que utilizem conhecimento de recursos da ferramenta, que os cursistas já adquiriram, como alteração de sons, ajuste de volume, animações, dentre outros, que foram abordados por meio de atividades e desafios durante o curso.

\section{Considerações Finais}

Ferramentas de linguagem de programação visual em blocos estão aos poucos integrando o dia a dia de professores na sala de aula. O Scratch é um exemplo destas ferramentas, permitindo a criação de jogos, animações e objetos de aprendizagem. No entanto, há outras ferramentas, como o AppInventor, que permite a criação de aplicativos para dispositivos móveis com o sistema operacional Android.

Uma vez que estudantes de diversas faixas etárias e professores assumem papel de desenvolvedores e projetistas de jogos e aplicações, torna-se então necessário que professores tenham conhecimento de recomendações de design, para que assim possam projetar interfaces adequadas para o público infantil, que na maioria das vezes consiste em seu público alvo. No entanto, isto não é uma tarefa trivial, uma vez que a percepção das crianças são muito diferentes da dos adultos, além da própria variabilidade que há entre crianças de idades distintas.

Este trabalho apresentou, de modo geral, recomendações para oito elementos de design. Um curso de formação de professores, que apresente estas boas práticas, pode contribuir para que a aplicação atinja os objetivos de usabilidade da interface, contribuindo para que o professor possa atingir seus objetivos em aula através do objeto de aprendizagem desenvolvido. Um curso está sendo planejado, onde pretende-se analisar os jogos desenvolvidos com base nas boas práticas de design levantadas neste trabalho.

\section{Referências}

Andrade, M., Neto, N., de Sousa, D. F., Chaves, R., \& Carvalho, G. (2015) “Análise dos Fatores Críticos para o Sucesso de Cursos de Graduação em Computação: um estudo de caso na Região Amazônica" In Anais do Workshop de Informática na Escola (Vol. 21, No. 1, p. 574). 
VI Congresso Brasileiro de Informática na Educação (CBIE 2017)

Anais do XXIII Workshop de Informática na Escola (WIE 2017)

Batista, E. J. S., Castro Jr, A., Cantero, S., Bogarim, C. A. C., e Larrea, A. A. (2016) "Uso do Scratch no ensino de programação em Ponta Porã: das séries inicias ao ensino superior" In Anais do Workshop de Informática na Escola (Vol. 22, No. 1, p. $565)$.

Batista, E. J. S., de Castro, C. P. C., \& de Castro, A. A. (2016) "Development of learning objects on scratch: Training of teachers of information and communication technologies" In Learning Objects and Technology (LACLO), Latin American Conference on (pp. 1-5). IEEE.

Bezerra, A. C. M. (2010) "Design da navegação em sites infantis educacionais: os efeitos no desempenho das tarefas", Tese da Pontifícia Universidade Católica do Rio de Janeiro.

Dalmoro, M., e Vieira, K. M. (2013) "Dilemas na construção de escalas Tipo Likert: o número de itens e a disposição influenciam nos resultados?" Revista gestão organizacional, 6(3).

da Silva, T. G., e Muller, F. M. (2012) "Jogos sérios em mundos virtuais: uma abordagem para o ensino-aprendizagem de teste de software" Master's thesis, Universidade Federal de Santa Maria.

Fabossi, L. A. e Guimarães, A. L. S. V. (2014) "Design de interface voltado a crianças em educação infantil” UTFPR faz pesquisa em design.

Hourcade, J. P. (2015) “Child-computer interaction”. Disponível em: http://homepage.cs.uiowa.edu/ hourcade/book/index.php. Acesso em 18 março de 2017.

Machado, L. S., Ronei M. M. e Nunes, F. (2009) "Serious games para saúde e treinamento imersivo." Abordagens Práticas de Realidade Virtual e Aumentada 1: 31-60.

Mano, A. (2005) "Interfaces de computador para crianças-avaliação e construção" Universidade do Minho.

Preece, J, Rogers, Y. e Sharp, H. (2013) "Design de interação: Além da interação humano-computador", 3. ed. Porto Alegre: Bookman.

Resende, C. A. T. D. S. (2010) "Design de interacção centrado nas crianças: estudo do caso Biblon" (Master's thesis, Universidade de Aveiro).

Sesame Workshop (2012) "Best practices: designing touch tablet experiences for preschoolers". Disponível em: http://www.sesameworkshop.org/assets/1191/src/Best\%20Practices\%20Document $\%$ 2011-26-12.pdf >. Acesso em 18 março de 2017.

Valente, J. F. P. (2011) “Avaliação da Usabilidade e Diversão em Interfaces Web para Crianças-caso de estudo escolinhas.pt" Mestrado Integrado em Engenharia Informática e Computação Tese de Mestrado Integrado em Engenharia Informática e Computação), Universidade do Porto, Faculdade de Engenharia. 